\title{
Long-Chain 3-Hydroxyacyl-CoA Dehydrogenase Deficiency
}

National Cancer Institute

\section{Source}

National Cancer Institute. Long-Chain 3-Hydroxyacyl-CoA Dehydrogenase Deficiency.

NCl Thesaurus. Code C129929.

An inherited condition caused by mutation(s) in the HADHA gene, encoding trifunctional enzyme subunit alpha, mitochondrial. It is characterized by hypoglycemia, hypotonia, neuropathy, cardiomyopathy, pig mentary retinopathy and may be associated with sudden death. 